\title{
TOWARDS A FAST, LOW-COST INDOOR MAPPING AND POSITIONING SYSTEM FOR CIVIL PROTECTION AND EMERGENCY TEAMS
}

\author{
E. Angelats ${ }^{\text {a, } * \text { J. A. Navarro }}{ }^{\text {a }}$ \\ ${ }^{a}$ Centre Tecnològic de Telecomunicacions de Catalunya (CTTC/CERCA), Av. Carl Friedrich Gauss, 7. Building B4, 08860 \\ Castelldefels, Spain - (eduard.angelats, jose.navarro)@cttc.es
}

Commission II

KEY WORDS: 3D modelling, Emergency response, Orientation, Rapid mapping, RGB-D camera, RPAS Photogrammetry

\begin{abstract}
:
Civil protection and emergency teams work usually under very risky conditions that endanger their lives. One of the factors contributing to such risks is the lack of knowledge about their physical environment, especially when working indoors. Mapping and location indoor and outdoor technologies exist; for outdoors, very good levels of precision and accuracy may be obtained using offthe-shelf equipment; on the other side, and although good solutions for indoor environments are available, these require some extra pre-deployed infrastructure in the area to navigate, which is unacceptable in the case of emergency teams. It may be said, then, that no mature indoor + outdoor integrated solution providing the appropriate precision and accuracy for the purposes of emergency teams exist. In this paper, the assessment of a set of currently available sensors (IMUs, RGB-D cameras, GNSS receivers) and algorithms is presented to show that it is already possible to build such a solution relying on them - providing that appropriate (indoor) lightning and texture conditions exist.
\end{abstract}

\section{INTRODUCTION AND MOTIVATION}

Civil protection and emergency teams working in areas affected by natural or man-made disasters - as for instance, floods, earthquakes or explosions - face challenging and risky situations that endanger their lives because of, among other reasons, the lack of knowledge about an environment, be it either indoor or outdoor, that may have changed drastically.

The authors believe that it is already possible to alleviate such situations developing a system able (1) to enhance the knowledge about the environment where these teams must work by means of quickly produced, low-cost indoor and outdoor cartography, (2) to continuously track the position of the members of the team using unobtrusive, portable positioning equipment based on RGB-D (Red, Green, Blue, Depth) cameras, Global Navigation Satellite Systems (GNSS) receivers and IMU (Inertial Measurement Unit) sensors, as well as sensor data fusion algorithms, and (3) to guarantee the communications among all the intervening components, either human or technological, by means of the deployment of an autonomous, portable communications network. Communications, however, are not be discussed in this paper; these are mentioned here in order to offer a complete picture of the concept presented by the authors.

Nowadays, the continuous location of emergency staff in indoor environments does not exist in practice. Of course, there exist both outdoor and indoor location solutions, but it is the integration of these in a seamless one what is missing, especially taking into account the needs of emergency teams. For instance, outdoor positioning based on single GNSS receivers or the hybridization of these and IMU sensors is rather common. Regarding indoor positioning, the lack of precise and accurate solutions is mainly due to the lack of GNSS signals in these environments. There exist, indeed, a few solutions providing seamless and continuous indoor and outdoor positioning, but these rely on the installation of devoted infrastructure deployed in advance. Such pre-deployment is, obviously, not possible when thinking of disaster management situations, since these may occur everywhere.

Algorithms for indoor positioning do exist; these are based on the fusion of imaging and IMU (and sometimes Light Detection and Ranging (LiDAR)) data. A simple integration of these data by means of the aforementioned algorithms in a lightweight, portable, battery-powered system-on-chip device would yield a very convenient indoor positioning system. The addition of a GNSS receiver will immediately enhance such device with precise outdoor positioning capabilities, opening the door to seamless positioning, no matter the whereabouts of the wearer indoors or outdoors. Obviously, such device must be light and unobtrusive so as not to hinder the already dangerous regular activities of the emergency team members.

Outdoor mapping is nowadays routinely performed by many companies using RPAS (Remotely Piloted Aircraft System), so obtaining quickly good quality outdoor cartography should be no problem at all; in fact, even the Copernicus Emergency Management Service offers a fast mapping service for emergencies using either Sentinel imagery or RPAS with a response time of about 48 hours - (CEMS, 2015; Ajmar, 2015) providing that local regulations allow it. But, as it happens with positioning, the problem of mapping is not so well solved when talking about indoor environments - especially when talking about areas that may have been severely affected by hazards, where part of the buildings may have collapsed.

It must be noted that, indeed, it is possible to obtain very good indoor cartography using well stablished technologies (as

* Corresponding author 
terrestrial LiDAR) using equipment manned by humans or carried by robots moving around (Kruijff-Korbayová, 2016). The problem is that this kind of solution may not be convenient when indoor mapping involves, for instance, working in buildings that may have partially collapsed or that may collapse at any moment due to some kind of disaster, thus endangering the live or lives of the operators. This is the reason why these techniques must be immediately avoided in these cases. Also, successful attempts to monitor indoor environments using terrestrial robots do exist (Kruijff-Korbayová, 2016). Nonetheless, the obstacles these robots may overcome are limited, being unable, for instance, to move between different floors in a building, which make these useless for the ultimate goal of this work. Assuming that after a disaster, parts of the building will have collapsed and that there may have appeared holes in the floors still reinforces this reasoning.

The approach presented in this paper to provide a solution for a safe procedure overcoming the aforementioned problems relies on the use of RPAS to generate indoor 3D cartography in confined spaces; obviously, not every RPAS will be suited for such task; for instance, fixed-wing ones must be immediately discarded, thus favouring multi-copters. In fact, models specifically designed to operate inside buildings (and thus, be resistant to blows and crashes) exist and have been successfully used for other purposes. These drones, equipped with the same set of sensors that the light positioning device mentioned above, are the solution conceived by the authors to solve the problem of indoor mapping.

Said that, the aim of this paper is to show that the technology and algorithms available nowadays are enough to build a lowcost fast 3D mapping and positioning system, specifically designed to fulfil the needs of civil protection and emergency teams. The accuracy and precision requested by emergency teams to operate in indoors environments needs not to be neither millimetric nor centimetric; in fact, being sure about what is the room they are in is enough for them. This implies that accuracies in the range of 1-2 metres and precisions between $30-50 \mathrm{~cm}$ are enough. This opens the way to build the lightweight, wearable positioning system as well as the payload carried by the drone to produce the $3 \mathrm{D}$ indoor maps of the buildings where such teams must work.

To do so, the relevant available sensors and algorithms will be presented and assessed. The limitations of these due to the work conditions present in the scenarios for which this concept is devised will also be discussed; for instance, the lighting conditions will hardly be optimal in interior environments after a disaster such as an earthquake or flooding because of the lack of light or presence of dust; this has a direct impact on the capabilities of some imaging sensors. The proposed architecture of the mapping and positioning system will also be presented ignoring, however, those aspects related to communications; only the mapping and positioning aspects will be taken into account.

\section{STATE OF THE ART}

\subsection{RPAS Mapping}

The rapid spread of the RPAS has led to significant price reductions and an exponential increase in their scientific usage, according to the number of scientific publications using RPAS as a mapping platform (Gomez and Purdie, 2016; Colomina and
Molina, 2014; Nex and Remondino, 2014). According to (Colomina and Molina, 2014) the decrease of price of the positioning, remote sensing and processors together with advances in the automation software have allowed their broad use. Nowadays, RPAS are able to carry out several mapping and positioning payloads such as cameras, laser scanner and GNSS receivers and inertial sensors (Giordan, 2017). RPAS or unmanned aerial vehicles $(\mathrm{UAV})^{1}$ can support hazard assessment and emergency management systems at both local and regional scale (Giordan, 2017), depending on the type (fixed wing, multicopters) of platform used (Gomez and Purdie, 2016; Boccardo, 2015). RPAS allow the mapping of land features and their evolution over time especially because of their rapid deployment. The work of (Gomez and.Purdie, 2016) presents a non-exhaustive review of UAV mapping for earthquakes, volcanic activity and landslides scenarios.

The Structure-from-Motion (SfM) approach (Schönberger and Frahm, 2016) is an efficient and robust technique for obtaining 3D models using RPAS platforms and COTS cameras (Nex and Remondino, 2014). The absolute scale and the proper geoferencing of the 3D models can be achieved using Ground Control, GNSS tagged images, or a combination of both. SfM is widely used, mostly because of the automation of the current photogrammetric software pipeline - as Pix4D (Pix4D, 2017), Agisoft (Agisoft, 2017) or Micmac (Pinte, 2017), among other and lower costs especially when compared to the RPAS LiDAR-based approach. Using GPU-enabled modern processors the required time to collect and process the data is significantly reduced (Remondino, 2017) leading to processing times of about a few hours, common in fast mapping for emergency scenarios. In the LiDAR-based approach, 3D models or DSMs are computed in real time combining laser raw measurements and the trajectory (positioning) of the platform. The trajectory errors have a direct impact on the geometric quality of the overlapping of point cloud strips. A postprocessing step is proposed (Glira, 2016) to improve the absolute and relative orientation, and thus properly registering these point clouds.

The use of RPAS for mapping in emergency scenarios is typical in outdoor environments and it is used as a valuable tool for fast mapping for civil protection and search and rescue teams (Boccardo, 2015; Kruijff-Korbayová, 2016; Dominici, 2017; Scaramuzza, 2014). On the contrary, the use of RPAS imagery for indoor environments is limited to research purposes; some works exist - in the context of NIfTi and TRADR projects, for the Emilia-Romagna (2012) and Umbria earthquakes (2016) providing aerial images from RGB cameras and 3D models generated in post processing (Kruijff, 2012; Kruijff-Korbayová, 2016) to assess building damages. Several indoor mapping experiences using RPAS also exist, but these are not targeted at the management of emergencies, such as the ones presented in (Mur-Artal and Tardós, 2017). (Mur-Artal and Tardós, 2017) propose a Simultaneous Localization and Mapping technique (SLAM), called ORB-SLAM, for the mapping problem. The proposed approach was tested with monocular, stereo and RGBD camera images.

\subsection{Indoor Positioning and Mapping}

Global Navigation Satellite Systems (GNSS) have been the tool for robust and precise positioning in the most recent times and

${ }^{1}$ For concision reasons and only in the rest of this section, the acronym RPAS will be used to talk about RPAS and UAVs. 
for the majority of applications. Yet, GNSS only works properly in good environment conditions (e.g., open air or clear lines-ofsight). GNSS performance drawbacks show up when operating, for instance, in urban or natural canyons or indoor environments. Then, the philosophy to overcome these limitations is based on setting a devoted infrastructure, such as visual beacons, ultra-wideband, Wi-Fi to mention a few, and having a system able to receive these signals to compute a solution using estimation algorithms (Mautz and Tilch, 2011; Dardari, 2015). This approach requires a number of reference nodes (anchor nodes or landmarks) deployed at fixed locations as well as one or more mobile nodes (receiver). Another approach is to combine the information provided by Inertial Measurement Unit (IMU) and aiding sensors such as cameras (monocular, stereo and RGB-D) and LiDAR sensors (Leutenegger, 2013; Veth, 2011).

Visual aiding refers to the computation of orientation parameters through consecutive, overlapping images by means of measurement of tie points -i.e., photogrammetric observations of the same object point in two or more images-. Extensive research and many algorithms have been proposed to robustly extract, describe and match common points, ideally invariant to orientation, scale and illumination changes (Lowe, 1999; Bay, 2008; Leutenegger, 2011). Feature extraction and matching algorithms are commonly combined, with RANSAC procedures that aim to perform outlier detection and removal using solely image observations by means of position and attitude estimation (Hartley and Schaffalitzky, 2004; Nistér, 2003). Other approaches combine derived trajectories or inertial data to predict where a point feature should appear in the second image (Veth, 2011; Taylor, 2011).

Once all outliers have been detected and isolated, the camera or vehicle trajectory may be recovered by concatenating the estimated relative positions and orientations using $k$ inliers from overlapping images. Several methods to estimate the navigation states using only images or using images and object observations are reviewed in (Hartley, 2000; Scaramuzza, 2011; Faessler, 2015; Forster, 2017). These approaches are usually referred as Structure from Motion (SfM) or visual odometry among the robotics and computer vision community. When a loop closure is detected and applied or a reference map is used to reduce positioning drift, visual odometry is known as SLAM. Alternatively, (Taylor, 2011) presents two strategies to use an IMU as a primary positioning sensor and to control the inertial drift with visual information during the filtering step. The two approaches are implemented using an Unscented Kalman Filter estimation method. The first approach imposes a geometric constraint using image coordinates while the second one takes benefit of jointly estimating a set of object coordinates together with navigation states.

\section{PROPOSED CONCEPT}

\subsection{Target Scenario and User Requirements}

The proposed system is targeted at emergency management and post-disaster assessment in the context of natural and man-made disasters that can cause severe building damage or collapse and loss of human lives. The considered emergency scenarios are: flooding, earthquakes, volcanic activities (eruption), landslides and subsidences, severe weather conditions (storms) and industrial accidents or damages to individual buildings due to explosions or fire (once the fire has been extinguished).
In these scenarios, the proposed system would be able to complement the current technologies and procedures, providing additional tools for a better emergency management. The use of these tools would even allow the improvement and / or adaptation of the emergency plans used during the intervention phase, increasing the safety of the staff as well as enhancing their coordination. Furthermore, the tools would serve to evaluate and increase post-disaster safety by assessing building damage, also contributing to faster building rehabilitation. The desirable aftermath would be to effectively reduce the number of victims in civil protection and emergency teams, by increasing the knowledge of their environment and reducing the risk of unexpected building collapse.

The goal would be to map the area affected by the disaster within a few hours. Mapping, in this context, means both outdoor and indoor mapping. Outdoor mapping is nowadays routinely performed by many companies using RPAS; even the Copernicus Emergency Management Service offers a fast mapping service for emergencies using either Sentinel imagery or RPAS with a response time of about 48 hours - providing that local regulations allow it. The cartography thus generated usually orthophotos - is more than enough for the purposes of the emergency teams ${ }^{2}$. Therefore, outdoor mapping will be treated in this paper as a problem already solved.

On the contrary, and as it happens with positioning, the problem of mapping is not so well solved when talking about indoor environments severely affected by hazards. Smoke or dust, for instance, is usually present after fires or earthquakes, thus impeding the normal operations of image sensors. In the context of this paper, proper lightning and texture conditions are assumed since the main sensor used to map indoors environments (see section 3.2) are RGB-D cameras. No assumptions on the ability to generate indoor mapping are made when these conditions are not met.

The outcome of indoor mapping in this context should be 3D models or 2D floorplans of the interior of the buildings affected by the disaster. The quality of these representations needs not to reach the usual geometric requirements found in other situations. In fact, emergency teams need to be reasonably sure about the room in which they are, as well as knowing which rooms are adjacent or if there is a way out or nearby holes in the floor or collapsed walls around. This kind of information may in fact save lives. Nonetheless, concerning precision and accuracy it is necessary to define a set of minimum requirements that, although pretty relaxed from the usual geometrical standpoint, would allow a representation of the buildings close enough to reality to be useful. These requirements are a few decimetres (between $30-50 \mathrm{~cm}$ ) for precision and a few meters $(1-2 \mathrm{~m})$ for accuracy.

Once the cartography describing the environment is available, it is desirable to be able to continuously locate the team members, either outdoors or indoors. In this way, it would be possible to know not only where they are, but also what risks threaten them or what escape routes are available. Nowadays the continuous location of emergency staff in indoor environments does not exist in practice. Concerning positioning requirements, a

\footnotetext{
${ }^{2}$ For instance, the Copernicus EMS Mapping is offering accuracies of one pixel and precisions of 5 pixels when the ground sampling distance is 1 meter or less. See (CEMS, 2015).
} 
precision of 0.5-2 meters will be enough in outdoors situations; for indoors, below 10 meters. Accuracy may not be assessed since there no reliable reference to compare to. Positions should be updated with a frequency of at least $1 \mathrm{~Hz}$.

\subsection{Mapping and Navigation Sensors. Processor}

The heart of the portable positioning device and the mapping payload will consist of an integrated GNSS + IMU module plus an RGB-D camera; these sensors will be driven by a SOC (System On Chip) taking care of all the data collection (including storage) and algorithmic tasks.

The GNSS + IMU module could be one such as the ublox NEO-M8U (ublox, 2017). This device delivers a complete position solution at $2 \mathrm{~Hz}$ when the GNSS signal is available. This would be the case of outdoor positioning. On the contrary, in GNSS-denied environments, that is, indoors in the context of this paper, the NEO-M8U delivers the linear accelerations and angular velocities from the IMU at $20 \mathrm{~Hz}$. This untethered dead reckoning behaviour perfectly matches the operational mode expected from the positioning device.

Section 3.1 states that proper illumination and texture conditions are some the requirements to fulfil for a system like this to work correctly. This is true, but it must be said that the technology of RGB-D cameras is evolving (and improving) continuously. Active RGB-D cameras $^{3}$ are able to measure depth even in 0 lux illumination conditions; The operational range is then noticeable reduced - that is, it is possible to measure depths only at closer ranges - and the precision and accuracy of the output point cloud is worse. Even though piloting an RPAS in 0 lux conditions may be impossible, this needs not to be this way when the situation is not so drastic. In other words, and providing that the illumination conditions allow the operation of the RPAS, active cameras may deliver data in situations where passive ones would not be able to do it. This is of utmost importance in emergency situations, where the availability of data may help to save lives. These two cameras would be good candidates to play expected role of the RGB-D sensor: the Intel ${ }^{\odot}$ Realsense $^{\mathrm{TM}}$ (Keselman et al., 2017) and Microsoft Kinect 2 (Lachat et al., 2015). However, the Kinect 2 should be discarded because of excessive power consumption issues (see section 4).

A lightweight, small footprint, low-power SOC is needed to assemble the sensors above and to provide the necessary computing power and storage to run the required algorithms and, in the case of the mapping payload, save the data that will be post processed at a later stage. Being lightweight and small is a very convenient property since the portable positioning device must be as unobtrusive as possible to avoid interfering with the activities of its wearers (the emergency staff). The low power consumption allows for a longer operational time before a battery replacement is needed. A powerful GPU is a desirable component due to the need to perform image processing computations on-the-fly (positioning device). The lack of such component may seriously compromise the performance of such device. The last generation of SOC provided by NVIDIA, the

\footnotetext{
${ }^{3}$ Active cameras, in this context, are those that emit infrared light to measure either a texture or a time-of-flight in order to compute depth. Passive cameras, on the contrary, use environmental light to do the same.
}

Jetson TX2 (Franklin, 2017) seem an appropriate candidate to assume this role.

Section 4 presents a feasibility analysis where the aforementioned sensors and related algorithms are assessed in a feasibility analysis.

\subsection{Operational Aspects}

The operation of the system, when working in indoors environments, is divided into three phases: (1) data collection, (2) 2D and 3D maps generation and (3) actual intervention of the emergency teams (with positioning).

Phase 1, data collection, is carried by a RPAS equipped with the mapping payload. While still located outdoors, this payload will compute the position and attitude of the RPAS using the GNSS receiver and the IMU module. The RGB-D cameras play no role at this moment. The position and attitude information thus obtained will be used as initial approximations as soon as the RPAS leaves the open space to go inside the affected buildings. Then, the mapping payload will not rely on the GNSS+IMU solution but on the RGB-D camera and IMU based solution. The images thus obtained will be stored together with their time tags. As soon as the RPAS returns to open spaces, the GNSS + IMU will be reactivated.

Note that the RPAS must face the challenge of flying in narrow, confined spaces presumably full of obstacles. Multicopters specifically adapted to fly in these circumstances exist and have been successfully used in other projects (Flyability, 2017).

In phase 2, the images captured by the drone are downloaded in the computer that will produce the $2 \mathrm{D}$ and $3 \mathrm{D}$ maps and models. See section (see section 3.4) for details about the software pipeline used to do that. It is worth to mention, however, that the use of RGB-D cameras implies the almost immediate availability ${ }^{4}$ of a coarse 3D model, made of the point clouds directly obtained by such cameras. Obviously, and since the orientation and position of the RGB-D images were estimated combining visual odometry and inertial data, their precision and accuracy will be worse than those obtained by the aforementioned pipeline. Nonetheless, and despite its lower quality, the availability of the coarse 3D map may be of crucial importance when a quick intervention of the emergency teams may imply saving lives; having coarse information about the environment where these teams must work is much more valuable than having no information at all.

In phase 3 the civil protection and emergency teams will enter the (indoor) affected areas, carrying the lightweight positioning device. Although not discussed in this paper, the availability of a communications module will allow to send the position of the staff to the control centre, so the management team will be constantly updated about where the personnel is located.

Last but not least, even if the lightning conditions degrademaking impossible the use of the positioning device - the maps and models previously generated are still very useful. The staff

\footnotetext{
${ }^{4}$ The time required to process the raw data coming from the RGB-D cameras and thus obtain a point cloud is almost negligible.
} 
at the control centre, in front of these models, may send indications to the emergency staff working inside the affected area using a regular communications link. Indications about the disposition of rooms, location of stairs, holes or collapsed walls are some of the examples of the information that may be provided to the emergency staff in this way.

\subsection{Software Pipeline}

When talking about software, two different cases must be told apart: positioning and mapping. The positioning software component is the heart of the portable positioning device carried by the members of the emergency team; the mapping component is the responsible for the generation of the 3D models.

\subsubsection{Positioning Software}

Once more, it is necessary to distinguish between two situations: positioning in indoor and outdoor environments.

The case of outdoors positioning is a classic one. In this situation, it is assumed that the GNSS signal will be available, so it will be used, together with the data collected by the IMU, to determine the position and attitude of the portable device by means of an extended Kalman filter / sequential least squares algorithm (this is, for instance, the approach in (Parés and Colomina, 2015). A global reference frame is used in this case.

When moving indoors, the portable positioning device will automatically switch to the second algorithm. In this case, the GNSS signal will not be available to visual odometry will be used instead. Again, an extended Kalman filter or a sequential least squares algorithm will be used. Data from the IMU will be used in the prediction step. The reference frame of the thus obtained orientation and position will be already a global one. In the filter step, the orientation and positions derived from the RGB-D images will be used to update the former prediction (as data coming from a GNSS receiver would have been used in outdoors situations), so the drift introduced by the IMU will be keep under control. It must be noted that the reference frame used by the position and orientation obtained from the images is a local one, but it is possible to switch to the global one using the lever arm and boresight matrices.

For these two data sources to work in tandem, it is necessary to use the same temporal reference frame - the internal clock of the processor of the portable positioning device is enough.

\subsubsection{Mapping Software}

The task of the mapping software is the offline generation of the 2D and 3D model of the area of interest. These are the required steps:

1. The whole set of data collected by the RPAS is downloaded to the computer responsible for the generation of the 2D and 3D model. This comprises GNSS (outdoors), IMU (both indoors and outdoors) and image data (indoors).

2. Coarse determination of initial approximations of the values of the positions and attitudes of the set of collected images. The algorithm used to do that is the one described in section (see section 3.4.1) for the positioning device.

3. Block adjustment to refine the aforementioned position and attitude data. There exist several tools able to perform this task, either open source or proprietary, as for instance Pix4D, AgiSoft or Micmac. This step is known as "Structure from motion" or "Integrated sensor orientation" depending on the technical community talking about it.

4. Generation of the dense point cloud, using the depth data (from RGB-D imagery) and the aforementioned position and orientation refined data. This algorithm to perform this task may be easily built using libraries as PCL (PCL, 2017).

\section{FEASIBILITY ANALYSIS OF THE CONCEPT}

There is a set of factors that must be taken into account when assessing the feasibility of the system proposed herein. These factors have implications in the selected hardware.

- Illumination and texture conditions. The use of active sensors (infrared emission) allows for very poor illumination (close to 0 lux) and texture conditions. The operating range directly depends on these conditions, as it happens to the ability to measure the depth and its quality. The quality of the indoor positioning techniques based on such sensors is therefore affected, since it relies on the capacity of extracting features from the RGB-D images (MurArtal and Tardós, 2017; Forster, 2017), however, shows the feasibility of the determination of the position in conditions of moderate illumination conditions.

- $\quad$ Time for real time processing, CPU load. Several works (Mur-Artal and Tardós, 2017; Forster, 2017) show the feasibility in terms of targeted output rate (positions for second) and CPU load of the proposed positioning techniques described in this paper using the suggested SOC (NVIDIA Jetson TX2).

- Time needed for mapping post-processing. The photogrammetric software packets available nowadays are able to produce results in reasonably short times, thus matching the requisites related to urgency and immediacy usually found in emergency scenarios (Remondino, 2017). Moreover, the volume of data to process is limited by the autonomy of the RPAS, which means that such volume will never be high; therefore, no big amounts of data need to be processed.

- Data synchronization issues. To work properly, the algorithms used for positioning or mapping require a proper time tag attached to all the data involved in the process. Furthermore, these time tags must be referred to the same temporal frame. High-quality products (in terms of accuracy and precision) normally require a devoted hardware providing such common time frame. This is not the case of the products (portable positioning device and mapping payloads) since the requested geometric quality makes possible to use the 
internal clock of the SOC processor. It would be possible, however, to characterize the drifts and latencies introduced by the system to improve a bit the temporal component of the system.

- $R G B-D$ cameras: influence of calibration and preheating time on the quality of the measurement of depth. Recent studies (Chesa, 2017; Keselman, 2017; Lachat et al. 2015) show that there is a series of factors that may produce systematic errors in the quality of the depth measurements and, consequently, in that of the derived point cloud used to generate the 3D model. These factors are an imperfect (or inexistent) geometric calibration of the camera, the pre-heating time and the low quality or the absence of texture in the areas of interest. However, the magnitude of these errors is negligible in the case of the application described herein, because the accuracy and precision requisites are sensibly lower than those affecting other more restrictive products.

- Power consumption and extra hardware or software requirements. The power consumption of some RGB$\mathrm{D}$ cameras is pretty high, which make these inadequate for the purposes of the system described in this paper. This is, for instance, the specific case of the Microsoft Kinect v2. Other models, including the Kinect v2, require extra hardware to work properly, delivering the expected performance (Chesa, 2017). Examples of these extra requirements could be the availability of a powerful GPU, CPUs compatible with OpenGL / OpenCL, or USB 3.0 connections. These constraints must be taken into account when selecting the camera.

- Extra cost versus photogrammetric improvements. Using an RGB-D camera instead of a regular RGB one may increase the cost of the system. On the other side, it has been shown (Mur-Artal and Tardós, 2017; Fang, 2015) that the solution obtained by means of RGB-D cameras is still robust in situations where the illumination conditions are poor or change quickly (e.g., contrast variations, shadows). Furthermore, the point cloud obtained directly from RGB-D cameras may be improved using post-processing techniques, using photogrammetric software packages, requiring very short times to deliver a useable 3D model.

\section{CONCLUSIONS}

This works presented a feasibility analysis of the technology and algorithms available nowadays to build a low-cost fast 3D mapping and positioning system, specifically designed to fulfil the needs of civil protection and emergency teams. The proposed concept has been designed to meet the accuracy and precision requested by emergency teams to operate in indoors environments. These requirements need not to be neither millimetric nor centimetric.

The reasons motivating this work have been presented, as well as the target scenarios where the proposed concept system would operate; particular emphasis has been put on defining the constraints regarding illumination and texture conditions, since these are typically the most important constraints to take into account. User requirements, specifically those related to accuracy and precision have been described and justified, since these are quite more relaxed than the usual geometric constraints found in other situations. Next, the sensors, algorithms and techniques needed to produce 3D maps and build a portable positioning device have been presented, together with a concept architecture for the system. Finally, a feasibility analysis closes this paper to show that a system to produce indoor cartography and a portable positioning device for emergency staff may be quickly integrated using the lowcost technology and algorithms presented in this work.

\section{REFERENCES}

Agisoft, 2017. Agisoft Photoscan. http://www.agisoft.com. Accessed: 20 October 2017.

Ajmar, A., Boccardo, P., Disabato, F. and Tonolo, F. G., 2015. Rapid Mapping: geomatics role and research opportunities. Rendiconti Lincei, 26(1), pp. 63-73.

Bay, H., Ess, A., Tuytelaars, T. and Van Gool, L., 2008. SURF: Speeded Up Robust Features, Computer Vision and Image Understanding (CVIU), 110(3), pp. 346-359.

Boccardo, P., Chiabrando, F., Dutto, F., Tonolo, F. G. and Lingua, A. (2015). UAV deployment exercise for mapping purposes: Evaluation of emergency response applications. Sensors, 15(7), pp. 15717-15737.

CEMS, 2015. Copernicus Emergency Management Service Mapping. Manual of Operational Procedures Guidelines for EC Services, Service Providers and Authorized Users. European Commission DG GROW, DG ECHO, DG JRC. Version 1.1 February 2015.

Chesa, M., 2017. Obstacle avoidance for an autonomous Rover. Bachelor Thesis, Technical University of Catalonia, 2017.

Colomina, I., and P. Molina. 2014. Unmanned aerial systems for photogrammetry and remote sensing: A review. ISPRS Journal of Photogrammetry and Remote Sensing, 92, pp. 7997.

Dardari, D., Closas, P. and Djuric, P, 2015. Indoor Tracking: Theory, Methods, and Technologies. IEEE Transactions on Vehicular Technology, 64(4), pp. 1263-1278.

Dominici, D., Alicandro, M. and Massimi, V., 2017. UAV photogrammetry in the post-earthquake scenario: case studies in L'Aquila. Geomatics, Natural Hazards and Risk, 8(1).

Faessler, M., Fontana, F., Forster, C., Mueggler, E., Pizzoli, M., Scaramuzza, D., 2016. Autonomous, Vision-based Flight and Live Dense 3D Mapping with a Quadrotor Micro Aerial Vehicle. Journal of Field Robotics, 33(4), pp. 431-450.

Fang, Z.; Zhang, Y., 2015. Experimental Evaluation of RGB-D Visual Odometry Methods. International Journal of Advanced Robotics Systems, 12, pp. 1-16. 
Flyability, 2017. ELIOS - Inspect \& explore indoor and confined spaces. www.flyability.com/elios, accessed 20 October 2017.

Franklin, D., 2017. NVIDIA Jetson TX2 delivers twice the intelligence to the edge. https://devblogs.nvidia.com/ parallelforall/jetson-tx2-delivers-twice-intelligence-edge, accessed 20 October 2017.

Forster, C., Zhang, Z., Gassner, M., Werlberger, M. and Scaramuzza, D., 2017. SVO: Semi-Direct Visual Odometry for Monocular and Multi-Camera Systems. IEEE Transactions on Robotics, 33(2), pp. 249-265.

Giordan, D., Manconi, A., Remondino, F. and Nex, F.C., 2017. Use of unmanned aerial vehicles in monitoring application and management of natural hazards. Geomatics, Natural Hazards and Risk, 8(1), pp. 1-4.

Glira, P., Pfeifer, N., Mandlburger, G., 2016. Rigorous Strip Adjustment of UAV-based Laserscanning Data Including TimeDependent Correction of Trajectory Errors. Photogrammetric Engineering and Remote Sensing, 82(12), pp. 945-954.

Gomez, C. and Purdie, H., 2016. UAV- based Photogrammetry and Geocomputing for Hazards and Disaster Risk Monitoring A Review. Geoenvironmental Disasters, 3(1), p. 1.

Hartley, R. and Zisserman, A., 2000. Multiple view geometry in computer vision. Cambridge University Press, 2nd edition.

Hartley, R. and Schaffalitzky, F., 2004. $L_{\infty}$ minimization in geometric reconstruction problems. Proceedings of Computer Vision and Pattern Recognition Conference, 1, pp. I-I.

Keselman, L., Iselin Woodfill, J., Grunnet-Jepsen, A. and Bhowmik, A., 2017. Intel ${ }^{\odot}$ RealSense $^{\mathrm{TM}}$ stereoscopic depth cameras. Proceedings of IEEE Conference on computer vision and pattern recognition workshops (CVPRW), pp. 1267-1276.

Kruijff, G.-J. , Tretyakov, V., Linder, T., Pirri, F, Gianni, M., Papadakis, P., Pizzoli, M., Sinha, A., Pianese, E., Corrao, S., Priori, F., Febrini, S. and Angeletti, S., 2012. Rescue robots at earthquake-hit Mirandola, Italy: A field report. IEEE Intl. Symp. on Safety, Security, and Rescue Robotics, pp. 1-8.

Kruijff-Korbayová, I. 2016. The Use of Robots for Disaster Response. Presentation at CTIF Delegates Assembly \& Symposium, Sep 8-9, 2016 in Helsinki.

Lachat, E., Macher, H., Landes, T. and Grussenmeyer, P., 2015. Assessment and calibration of a RGB-D Camera (Kinect v2 sensor) towards a potential use for close-range 3D modeling. Remote Sensing, 7(10), pp. 13070-13097.

Leutenegger, S., Chli, M. and Siegwart, R., 2011. BRISK: Binary Robust Invariant Scalable Keypoints. Proceedings of the IEEE International Conference on Computer Vision (ICCV), pp. 2548-2555.

Leutenegger, S., Furgale, P.T., Rabaud, V., Chli, M., Konolige, K. and Siegwart, R., 2013. Keyframe-Based Visual-Inertial SLAM using Nonlinear Optimization. Proceedings of Robotics: Science and Systems 2013.
Lowe, D., 1999. Object recognition from local scale-invariant features. Proceedings of the International Conference on Computer Vision (ICCV), 2, pp. 1150-1157.

Mautz, R. and Tilch, S., 2011. Survey of Optical Indoor Positioning Systems. Proceedings of the 2011 International Conference on Indoor Positioning and Indoor Navigation (IPIN), Guimarães, Portugal, 21-23 September, 2011.

Mur-Artal R., and Tardós, J.D, 2017. ORB-SLAM2: an OpenSource SLAM System for Monocular, Stereo and RGB-D Cameras. IEEE Transactions on Robotics, 33(5), pp. 12551262.

Nex, F.C., and Remondino, F., 2014. UAV for 3D mapping applications: a review. Applied Geomatics, 6 (2014)1 pp. 1-15.

Nistér, D., 2004. An efficient solution to the five-point relative pose problem. IEEE transactions on pattern analysis and machine intelligence, 26(6), pp. 756-770.

Parés, M.E. and Colomina, I., 2015. On software Architecture Concepts for a Unified, Generic and Extensible Trajectory Determination System. Proceedings of the ION GNSS+, 08-12 September 2015, Tampa, Florida (USA).

Pinte, A., 2017. Micmac, un logiciel pour la mise en correspondance automatique dans le contexte géographique. http://logiciels.ign.fr/?Micmac, accessed 20 October 2017.

Pix4D, 2017. Pix4D - Drone photogrammetric software for desktop + cloud + mobile. https://pix4d.com, accessed 20 October 2017.

Remondino, F., Nocerino, E., Toschi, I., and Menna, F, 2017. A Critical Review of Automated Photogrammetric processing of large datasets. The Int. Arch. Photogramm. Remote Sens. Spatial Inf. Sci., XLII-2/W5, pp. 591-599.

Scaramuzza, D. and Fraundorfer, F., 2011. Visual Odometry [Tutorial]. IEEE Robot. Automat. Mag., 18(4): 80-92.

Scaramuzza, D., Achtelik, M. C., Doitsidis, L., Friedrich, F., Kosmatopoulos, E., Martinelli, A., ... and Gurdan, D., 2014. Vision-controlled micro flying robots: from system design to autonomous navigation and mapping in GPS-denied environments. IEEE Robotics \& Automation Magazine, 21(3), pp. 26-40.

Schönberger, J. L., and Frahm, J.M., 2016. Structure-fromMotion Revisited. Proceedings of IEEE Conference on Computer Vision and Pattern Recognition (CVPR), pp. 41044113.

Taylor C.N, Veth M.J., Raquet J.F., Miller M.M, 2011. Comparison of Two Image and Inertial Sensor Fusion Techniques for Navigation in Unmapped Environments. IEEE Transactions on Aerospace and Electronic Systems, 47(2), pp. 946-958.

ublox, 2017. NEO-M8U module. https://www.ublox.com/en/product/neo-m8u-module, accessed 20 October 2017.

Veth, M.J., 2011. Navigation Using Images, A survey of Techniques. Journal of Navigation, 58(2), pp. 127-140. 